\title{
Belts Evaluated as Limb Tourniquets: BELT Study Comparing Trouser Supporters Used as Medical Devices in a Manikin Model of Wound Bleeding
}

\author{
Blake W. Bequette, BS; John F. Kragh Jr, MD; James K. Aden 3rd, PhD; Michael A. Dubick, PhD \\ From the United States Corps of Cadets, United States Military Academy, West Point, NY (Mr Bequette); and the United States Army Institute of \\ Surgical Research, Joint Base San Antonio Fort Sam Houston, TX (Drs Kragh, Aden, and Dubick).
}

\begin{abstract}
Objective.-The purpose of the present study is to compare several models of commercially designed belts as used as a tourniquet.

Methods.-In the Belts Evaluated as Limb Tourniquets (BELT) study, an experiment was designed to test the effectiveness of pants belts as nonimprovised medical devices to control hemorrhage in a manikin. Models of belts included Tourni-belt, Tourniquet Belt, ParaBelt, and Battle Buddy. Data collected included effectiveness, time to stop bleeding, total time of application, pressure, blood loss, and composite results (score count of good results; composite outcome good if every component was good).

Results.-Differences in effectiveness percentages among models were not statistically significant. The difference in mean between users was statistically significant for stop time, total time, pressure, blood loss, composite score, and composite outcome. Mean time to stop bleeding differed for only 1 pair of models after the Tukey-Kramer adjustment; ParaBelt was faster than Tourniquet Belt. Mean total time of application differed between ParaBelt-Tourniquet Belt and Tourni-belt-Tourniquet Belt; the former model in both pairs was faster. No significant difference in mean blood loss measured by model was found. For composite outcome score, no pairwise difference between models was significant. For composite outcome (good-bad), ParaBelt had good results in $75 \%$ of tests; the other 3 models had significantly worse results.

Conclusions. - In a preliminary laboratory analysis of belt tourniquet models using a manikin, performance differed by model. ParaBelt performed better than other models for the composite outcome.
\end{abstract}

Keywords: first aid, emergency medical services, medical device, resuscitation, shock, hemorrhage/ prevention and control, tourniquet

\section{Introduction}

Traumatic wounds can result in severe bleeding that, if not controlled, may become lethal. ${ }^{1-3}$ Control of bleeding from limb wounds via tourniquet use has been shown to be reliably effective and potentially lifesaving. ${ }^{4-8}$ Commercially designed trouser belts have been intentionally made so that they are readily available to serve a

Corresponding author: John F. Kragh, Jr, MD, US Army Institute of Surgical Research, 3698 Chambers Pass, JBSA Fort Sam Houston, TX 78234-7767; e-mail: john.f.kragh.civ@mail.mil.

Submitted for publication May 2016.

Accepted for publication January 2017. second role as a tourniquet when needed to prevent and control bleeding from traumatic wounds. In these 2 ways, belt tourniquets are dual-purpose. A belt tourniquet is both a medical device and an article of clothing because it can be worn as a traditional belt with pants and taken off for application as a limb tourniquet if needed. Such tourniquets are not improvised because they are specifically designed for use as a tourniquet are also designed as a band to support trousers at the waist. To achieve required pressure to stop blood flow, a tourniquet uses a mechanical advantage such as a windlass, pulley, or ratchet.9,10 By tightening around a limb, a tourniquet stops the 
underlying arterial blood flow to control bleeding from distal wounds. ${ }^{8}$ However, public awareness of such medical devices is limited because they are new and have not yet been widely fielded to military servicepersons or law enforcement officers.

Recent scientific discussions in the medical literature about tourniquets have been many, but the topic of belt tourniquets is new. Currently, few data are available on the effectiveness of belt tourniquets. A better understanding of the usefulness of these devices could inform their design and development or decisions on use in the field, such as by soldiers, outdoorsmen, or police officers. A laboratory assessment of a human-like manikin with a limb wound in need of hemorrhage control would be useful in delivering performance data and possibly improving awareness. The purpose of this study was to assess the differential performance of 4 models of belt tourniquets regarding their capacity to control hemorrhage.

\section{Methods}

A US Army Institute of Surgical Research laboratory protocol was approved by the Institute's Surgical Research's Regulatory Compliance Division. A biomechanical experiment was conducted with a manikin and using Belts Evaluated as Limb Tourniquets (the BELT study).

Four models of belt tourniquets were studied. Model designs included 1 using a pulley, 1 using a ratchet, and 2 using a windlass (Table 1; Figure 1). The US Food and Drug Administration (FDA) has classified such devices as nonpneumatic tourniquets. A nonpneumatic tourniquet is defined as a device consisting of a strap or tubing intended to be wrapped around a patient's limb and tightened to reduce circulation (regulation 878.5900). ${ }^{11}$ Such a tourniquet is device class 1 (as defined in 21 Code of Federal Regulations Parts 862 to 892) within the medical specialty of general and plastic surgery. Although nonpneumatic tourniquet devices are exempt from 510(k) criteria in submission to FDA, they are not exempt from good manufacturing practices.
The first model, Tourni-belt (Special Designs, La Vernia, TX), had a strap-and-windlass design. The strap was woven from cord $(250 \mathrm{~kg}$ [550 pound] test parachute cord or type-III paracord) in 2 layers. Such cord is a lightweight rope with a nylon kernmantle, consisting of an interior core (kern) and woven exterior sheath (mantle), which is used in the suspension lines of a parachute. As woven, the configuration of the Tournibelt had an inner layer of 2 cord segments running longitudinally inside an outer layer of woven cord. Turning the windlass twisted the inner cords around the center of the windlass. Turning the windlass tensioned the inner cords, which also mechanically loaded the adjacent outer layer. The windlass was an aluminum handle, which provided leverage in the mechanical application of tightening. The device did not have a clip to secure the windlass after its winding. The belt length overall is $1070 \mathrm{~mm}$ with a width of $40 \mathrm{~mm}$. The model tested was a prototype that was not yet registered with the FDA at the time of assessment.

The second model, the Tourniquet Belt (Rapid Application Tourniquet [RATS] http://ratstourniquet.com), also had a strap-and-windlass design. The strap was also in 2 layers. The inner layer was also made of 2 cords (550pound test parachute cord) running longitudinally inside an outer layer. The outer layer was made of nylon webbing in 2 laminas between which lay the 2 cords of the inner layer. The windlass also was an aluminum handle. A cobra buckle in 2 parts, 1 at each end of the strap, snaps together a male end and a female end so that the strap goes around the limb circumferentially. The RATS Tourniquet belt (US patent 8343182) looks like a regular tactical belt, first line belt, or pistol belt, but it is also an available tourniquet when needed. The belt comes in 12 lengths, in $5-\mathrm{cm}$ increments from $55 \mathrm{~cm}$ to $111 \mathrm{~cm}$. The tested belt length overall is $1100 \mathrm{~mm}$ with a width of $38 \mathrm{~mm}$. The device assessed was in coyote color, a medium brown hue, but black is also available. The model tested was a prototype and was registered with the FDA.

The third model of belt tourniquet was ParaBelt Tourniquet Belt (RevMedx, Wilsonville, OR). The belt is made of black nylon webbing as a daily-wear belt

Table 1. Belt tourniquet traits by model

\begin{tabular}{lcccll}
\hline Model of belt tourniquet & Width $(\mathrm{cm})$ & Length $(\mathrm{cm})$ & Weight $(\mathrm{gm})$ & Cost $($ USD $)$ & Mechanical advantage \\
\hline Tourni-belt & 3.5 & 117 & 280 & N/A & Windlass \\
Tourniquet Belt & 3.9 & 110 & 134 & 99 & Windlass \\
ParaBelt & 4.5 & 120 & 240 & 125 & Ratchet \\
Battle Buddy & 4.5 & 112 & 261 & N/A & Two pulleys \\
\hline
\end{tabular}

USD, US dollars; N/A, not applicable, no sales currently. 
A

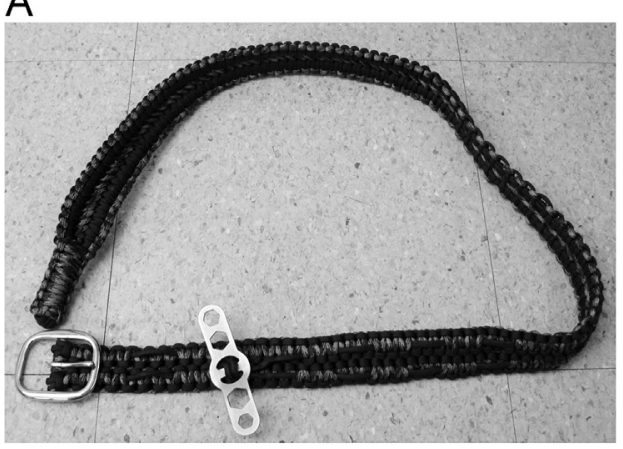

C

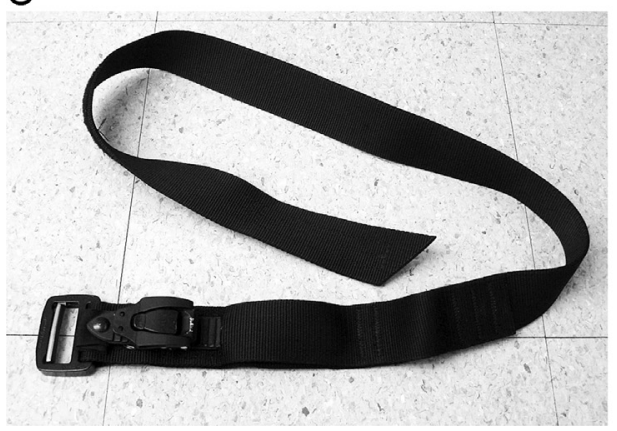

B

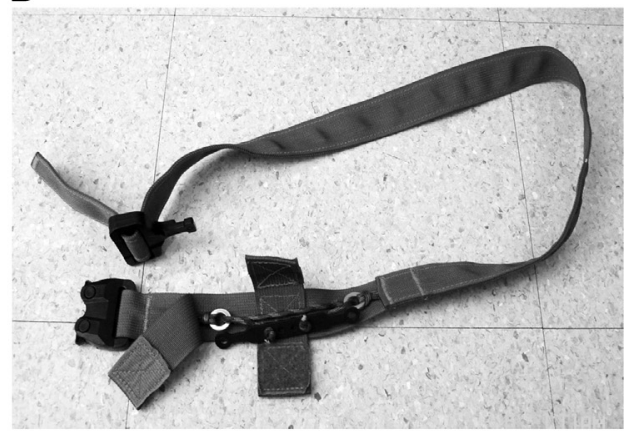

D

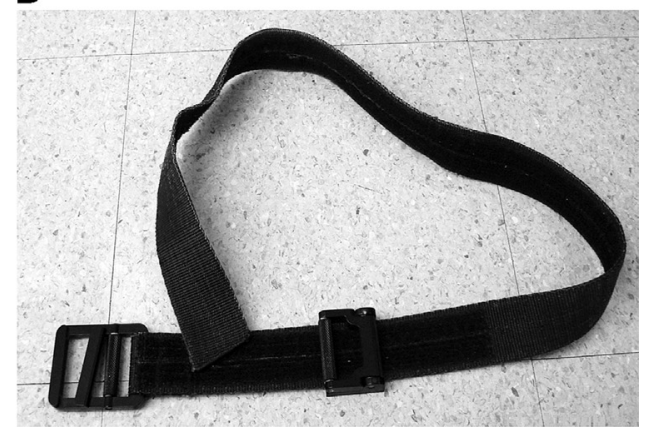

Figure 1. (A) Photograph of Tourni-belt, a strap-and-windlass design. (B) Photograph of Tourniquet Belt, a strap-and-windlass design. (C) Photograph of ParaBelt, a ratchet design. (D) Photograph of Battle Buddy, a pulley design.

with an integrated tourniquet of a ratchet design. The ratchet was similar to those of outdoor sporting equipment (eg, boot buckles for roller blades or snowboard bindings) so those with experience with such ratchets may find the design simple, if not intuitive, to operate. ParaBelt is nearly as compact and lightweight as a tactical belt, and its components are made to military specification with regard to elements such as durability. The marketing targets first responders, law enforcement, outdoor enthusiasts, and concerned citizens. The belt length overall is $1200 \mathrm{~mm}$ with a width of $43 \mathrm{~mm}$. The model tested was registered with the FDA (reg. 3009035955).

The fourth model, Battle Buddy (Hawaii Product Development, Honolulu, HI), has a pulley design: Two separate pulleys aid in tightening the belt. The belt is looped through the slots in the pulley so that the belt is tightened as it rolls over the pulley wheel to reverse the direction of pull. Such pulling of the belt through the pulley removes slack from the belt tourniquet. Additional pulling of the belt through the second pulley gains is in the opposite direction of pull from the first pulley. Mechanical advantage is gained from use of the 2 pulleys. Additionally, the second pulley wheel is offset away from the belt (away from the leg) to increase the moment arm and torque of the pull force. The belt length overall is $1100 \mathrm{~mm}$ with a width of $44 \mathrm{~mm}$. The device
(US patent $0215254 \mathrm{~A} 1$ ) tested was not registered with the FDA at the time of assessment.

Two persons tested performance of the tourniquets. One user (JFK) was a clinician-scientist who was an expert in tourniquet application, with experience including trauma care, research, and development. The other user (BWB) was a US Military Academy cadet with no medical experience, but who had been trained in first aid use of the limb tourniquet, which was standard issue in the military service. Data were collected from May 21 to June 8 in 2015.

One-hundred sixty tests were conducted. There were 2 users, 4 models of belt tourniquets, and 20 tests of each model per user. Each user's testing consisted of 20 blocks of tests; each block consisted of a single test of 1 tourniquet of each of the 4 models. Because tourniquet designs were similar for some models, within each block, the test order was randomized by model to mitigate user effects like differential learning by tourniquet design.

The tourniquets were tested on a laboratory manikin (HapMed Leg Tourniquet Trainer, CHI Systems, Fort Washington, PA) that was designed to train users by providing feedback on user performance; the right thigh had an above-the-knee amputation injury. ${ }^{12-16}$ An embedded computer had a smartphone-like touchpad integral to the thigh (software version 1.9, CHI Systems, 
Fort Washington, PA). The manikin was operated by user input through finger touch on the pad. The manikin's thigh was placed on a laboratory bench and was operated in accordance with the manufacturer's instructions. The touchpad was lighted during tests, showing the runtime feedback screen to provide users with visual information on time, pressure, and bleeding control.

The thigh did not bleed; rather, bleeding was represented by red lights that transilluminated the wound. The number of lights represented the bleeding rate: all 26 lights being lit meant maximal bleeding; no lights meant bleeding had stopped; and a few lights twinkling indicated intermediate control. Arterial pulses were palpable in the popliteal area. Touchpad readouts included hemorrhage control, the time of application, total time of the trial (including assessment time), the pressure exerted under the tourniquet, and the simulated blood loss volume. Iterations began with the tourniquet laid out undone (unpackaged, folded, flattened, and stretched out length-wise) on the bench and not yet applied to the thigh. The time of application was designated to be the time interval between the beginning of the iteration and the time point at which the manikin detected that no more blood was lost. Once the manikin detected the cessation of blood loss, the tourniquet was considered to be effective. In cases in which the user could not make the tourniquet effective despite repeated attempts, the user terminated the test; these results were included as failed or ineffective tests. For ineffective tests, the time of termination was used as the time to stop bleeding, which equaled the time of application in such tests. The casualty had a medium build, and the setting was care under fire, an emergency situation wherein the casualty and caregiver are in danger (eg, ongoing gunfire).

The manikin settings also included a constant hemorrhage rate $(635 \mathrm{~mL} / \mathrm{min})$. The resulting bleed-out time was 4 minutes to apply the tourniquet successfully if there was no hemorrhage control at all until the end. Periods with intermediate hemorrhage control, even interspersed among periods with no hemorrhage control, could yield longer bleed-out times before the casualty status became "dead".

Using the data gathered, additional results were calculated as the composite results of 5 other scores, which included hemorrhage control, time to stop bleeding, total time of application (includes time of assessing the casualty), the pressure exerted under the tourniquet, and the simulated blood loss volume. The composite results were made in 2 ways. The first was a composite score for each test as a number. The count (0 to 5) of the 5 possible elements that were satisfactory in each test were 1) that hemorrhage control was yes; 2) the time of to stop bleeding was $\leq 60$ seconds; 3 ) the pressure was within $150 \mathrm{~mm} \mathrm{Hg}$ and $300 \mathrm{~mm} \mathrm{Hg}$, inclusive; 4) blood loss was $<500 \mathrm{~mL}$; and 5) no breakage of any tourniquet component occurred. The second composite outcome was a binary aggregate as either a "good" or "bad" result for each test, whether or not every single element making up the composite was satisfactory.

During the data collection, both users recorded their own subjective assessments of the models of belt tourniquet to supplement the quantitative assessments. Design traits of belt tourniquets were considered as relative advantages or disadvantages by model.

In the statistical model, the model of tourniquet was considered the treatment for which a treatment effect was sought on outcomes. If there was a clear user effect (a nontreatment effect), a mixed model analysis of variance (ANOVA) was planned additionally, with user being a random effect in the mixed statistical model. All pairwise comparisons were adjusted using the TukeyKramer method; 4 models had 6 pairings for comparison. A value of $P<.05$ was considered significant.

\section{Results}

\section{RESULTS OF USER EFFECTS}

Users were not statistically different in effectiveness of use of tourniquet belts for bleeding control $(P=.06)$ and for cessation of popliteal pulse $(P=.1)$. However, user differences were significant in the remaining performance categories. The difference between users in mean time to stop bleeding was statistically significant $(25$ seconds, $P=.002$ ). Likewise, users differed in mean total time of application (19 seconds, $P=.01$ ), tourniquet pressure $(24 \mathrm{~mm} \mathrm{Hg}, P<.0001)$, blood loss volume $(25 \mathrm{~mL}, P<.0001)$, composite score $(0.56$, $P<.0001)$, and composite outcome $(34 \%$ in good results, $P<.0001)$. Compared with the expert, in all these differences, the performance of the cadet was better for time to stop bleeding, total time of application, pressure, blood loss, and both composite results. The user effect constituted $24 \%$ of the estimated variance within the statistical model. Because of the significance of these several user effects, further statistical analysis was undertaken with the planned mixed statistical model with ANOVA.

\section{RESULTS OF EFFECTIVENESS BY TOURNIQUET MODEL IN THE MIXED STATISTICAL MODEL}

Results of ANOVA for effectiveness (bleeding control, yes or no) by model was in 1 tier; effectiveness percentage by model did not differ by model of belt 
Table 2. Performance results by model of belt tourniquet

\begin{tabular}{lccc}
\hline Belt tourniquet Model & Time to stop bleeding (mean $\pm S D) s$ & Blood loss (mean $\pm S D)$ mL & Composite outcome: good (\%) \\
\hline & $67 \pm 6$ & $348 \pm 32$ & 45 \\
Tourni-belt & $85 \pm 6$ & $363 \pm 21$ & 33 \\
Tourniquet Belt & $56 \pm 6$ & $352 \pm 24$ & 75 \\
ParaBelt & $80 \pm 12$ & $361 \pm 50$ & 50 \\
Battle Buddy & &
\end{tabular}

Results of mean time to stop bleeding by model were in 2 tiers. The fast tier included ParaBelt, Tourni-belt, and Battle Buddy, and the slow tier included Tourniquet Belt, Tourni-belt, and Battle Buddy. With ANOVA, a tier comprises the means of models that are not statistically significant in their difference, whereas differences between means of tiers are significant, except for means that are in $>1$ tier because a mean cannot be different from itself. The only pairwise comparison that differed was ParaBelt-Tourniquet Belt $(P=.048)$. Differences in mean blood loss were not significant. ParaBelt had good results more often than other models $(P=.001)$. SD indicates standard deviation.

tourniquet $(P=.2)$ as effectiveness ranged from a minimum of $93 \%$ to a maximum of $100 \%$ for Tourniquet Belt and Battle Buddy, respectively. For both users, all 4 models were frequently successful in bleeding control (94\% and $100 \%$ for the expert and cadet, respectively, $p=0.2$ ) and pulse cessation (95\% and $100 \%$ for the expert and the cadet, respectively, $P=.4$ ).

\section{Results of Time to Stop Bleeding by Model}

Results of ANOVA for mean time to stop bleeding by model were in 2 tiers. The fast tier had means of 56 seconds for ParaBelt, 67 seconds for Tourni-belt, and 80 seconds for Battle Buddy, whereas the slow tier included Tourniquet Belt (mean, 85 seconds), Battle Buddy, and Tourni-belt. In Tourniquet Belt use, bleeding was stopped, on average, 1.5 times slower than in ParaBelt use (Table 2, Figure 2).

In pairwise comparison, mean time to stop bleeding differed for only 1 pair of models after the TukeyKramer adjustment: Tourniquet Belt (85 seconds) and ParaBelt (56 seconds, $P=.048$; all 5 other pairs [Tourni-belt-Battle Buddy, Tourni-belt-Tourniquet Belt, Tourni-belt-ParaBelt, Tourniquet Belt-Battle Buddy, and Battle Buddy-ParaBelt], $P \geq .1$ ).

\section{RESULTS OF TOTAL TIME OF APPLICATION BY MODEL}

Results of ANOVA for mean total time of application by model were in 2 tiers. The fast tier included ParaBelt, Tourni-belt, and Battle Buddy with means of 73 seconds, 82 seconds, and 100 seconds, respectively, and the slow tier included Tourniquet Belt and Battle Buddy. With ANOVA, a tier comprises the means of models that are not statistically significant in their difference; differences in means between tiers is significant, except for those means that are in $>1$ tier because a mean cannot be different from itself. All means exceeded 60 seconds.
In pairwise comparison, mean total time differed for 2 pairs of models: ParaBelt-Tourniquet Belt $(P=.0001)$ and Tourni-belt-Tourniquet Belt $(P=.003)$ (all 4 other pairs, $P \geq .09$ ).

\section{RESULTS OF PRESSURE BY MODEL}

Results of ANOVA for mean pressure by model were in 2 tiers. The high tier included ParaBelt, Tourni-belt, and Battle Buddy, with means of $195 \mathrm{~mm} \mathrm{Hg}, 195 \mathrm{~mm} \mathrm{Hg}$, and $189 \mathrm{~mm} \mathrm{Hg}$, respectively. The low tier included Tourniquet Belt (mean, $159 \mathrm{~mm} \mathrm{Hg}$ ). All means ranged between $150 \mathrm{~mm} \mathrm{Hg}$ and $200 \mathrm{~mm} \mathrm{Hg}$.

In pairwise comparison, mean pressures applied by belt tourniquet models differed only for Tourni-belt paired with each of the other 3 models $(P \leq .0003$, all 3).

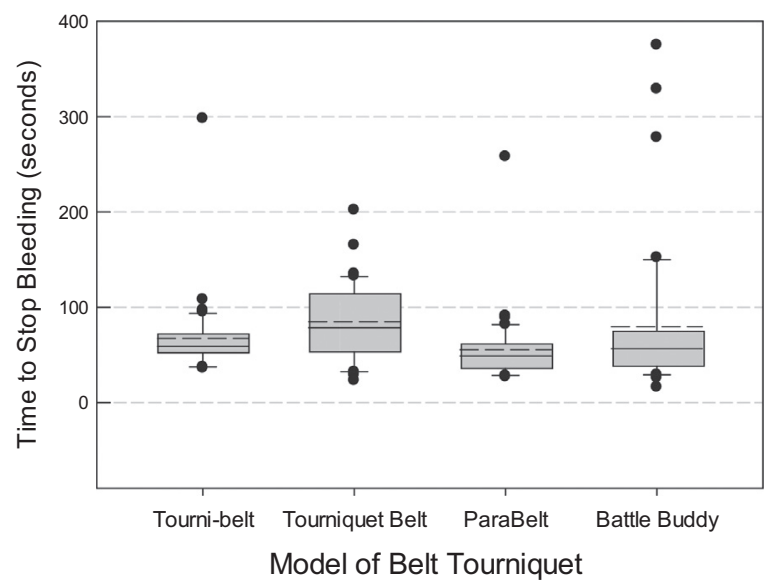

Figure 2. Vertical box plots have bottoms as 25 th percentile, tops as 75th percentile, the solid line as the median, the dashed line as the mean, the up bar as 95th percentile, the down bar as the 5th percentile, and the dots as data points beyond the bars. The fast tier of results included the ParaBelt, Tourni-belt, and Battle Buddy, and the slow tier included the Tourniquet Belt, Battle Buddy, and Tourni-belt. The mean time to stop bleeding differed for only 1 pair: Tourniquet Belt and ParaBelt $(P=.048)$. 


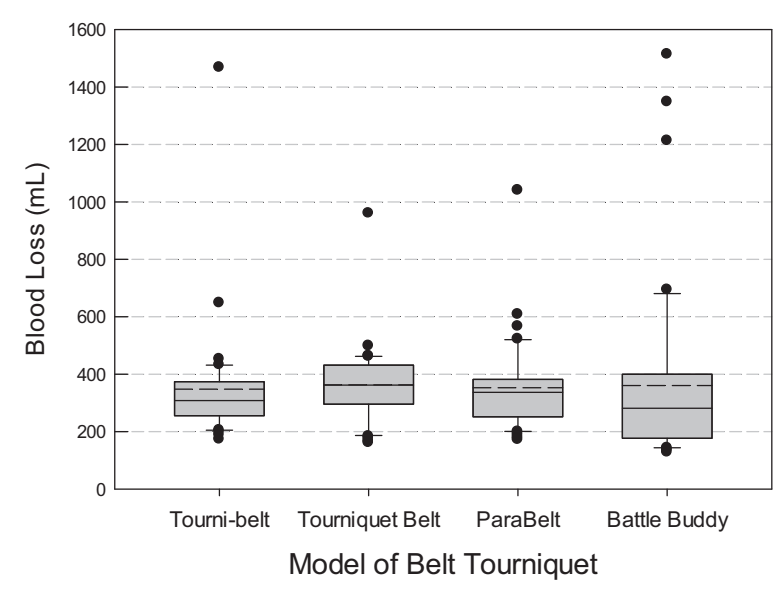

Figure 3. There was no significant difference in mean blood loss measured among the models.

\section{RESULTS OF BLOOD LOSS BY MODEL}

Results of ANOVA for mean blood loss by model were in 1 tier. There was no significant difference in mean blood loss $(P>.9$, all; range $348-363 \mathrm{~mL}$; Table 2, Figure 3).

\section{COMPOSITE RESULTS BY MODEL}

The results of ANOVA for composite score, as a count of the 5 data elements that were satisfactory for each test, were in 1 tier. Models did not differ significantly $(P \geq$ .09). All models had a mean composite score within a narrow range between 4.2 and 4.6 (between Tourniquet Belt and ParaBelt, respectively). Furthermore, no pairwise difference was significant ( $P \geq .09$, all 6 pairs).

The results of ANOVA for composite outcome (good or bad) were in 2 tiers. ParaBelt was in the high tier, and all 3 others were in the low tier. ParaBelt had good results in $75 \%$ of tests, whereas Tourniquet Belt, Tournibelt, and Battle Buddy had good results in $33 \%, 45 \%$, and $50 \%$ of tests, respectively $(P=.001$; Table 2$)$.

\section{RESULTS OF SAFETY-RELATED OBSERVATIONS AND SUBJECTIVE ASSESSMENTS}

During testing, the ParaBelt had mechanical wear of the ratchet-pawl mechanism. In its design, the flat band (plastic or polymer) acts as the ratchet and is notched transversely like a ladder so that the pawl fits into the notches. The pawl is a metal wheel with multiple teeth, which fit into the ratchet notches. The wear occurred in the ratchet at the tips between the notches when a pawl tooth slipped over a tip, thereby rounding off the normally sharp point of the tip. The only device that had such wear had it occur on 2 adjacent tips. This device was removed from testing after completion of the test in which the damage occurred, and that device was replaced with a new one. Wear was detected in only this 1 test, but the user was able to achieve the minimal pressure to stop bleeding, albeit after a lengthy, arduous effort.

The manikin's damage was limited to routine wear and tear of the silicone skin, which is about $8 \mathrm{~mm}$ thick. Regarding patient safety, 1 user noted that he thought 2 models, the Tourni-belt and Battle Buddy, looked like they may be more uncomfortable in use on patients. User subjective assessments of the models of belt tourniquet included the identified advantages and disadvantages for each model (Table 3).

\section{Discussion}

The main finding of the present BELT study, a very first look at this subject, was that ParaBelt performed best among the 4 models of belt tourniquet within the limited ways it was tested. In a preliminary analysis of belt tourniquet models in a laboratory setting using a manikin, performance differed by model. The ParaBelt model had a higher percentage of tests with good results than the other 3 models. Furthermore, subjective results were complimentary to and coherent with the quantitative assessments. The belt models of tourniquets studied presently were purposefully designed as medical devices, that is, primarily to work as tourniquets and also to function as belts and not vice versa. The newness of the 4 models, the newness of nonimprovised belt tourniquets, and the newness of the present study's findings may aid in improving awareness of belt tourniquets available today. The wider diversity of designs of tourniquet models that have become available since 2001 provides the community with more choices, but also more needs to validate function, and the present study provides a measure of differential function of 4 belt tourniquet models available in 2015 .

The first minor finding of the present study was that tourniquet data in bleeding control continue to show substantial effects from the user, a nonrandom, nontreatment effect within the statistical model. The user effect constituted $24 \%$ of the estimated variance within the statistical model. User differences were significant in most performance categories, such as time to stop bleeding, pressure, and blood loss. The cadet outperformed the expert in all these results. The cadet implicitly took a strategy of optimizing performance, while the expert, an experienced researcher of tourniquet use, implicitly took a strategy of optimizing knowledge generation. The expert allowed himself to use trial and error to assess variant techniques of tourniquet model use 
Table 3. Advantages and disadvantages by model of belt tourniquet based on subjective assessments of users

\begin{tabular}{|c|c|c|}
\hline Model of belt tourniquet & Advantage & Disadvantage \\
\hline Tourni-belt & $\begin{array}{l}\text { Windlass mechanism is } \\
\text { intuitive and reliable } \\
\text { Minimum mean blood loss }\end{array}$ & $\begin{array}{l}\text { Limited tightening before windlass use } \\
\text { Occasional pinching of skin }\end{array}$ \\
\hline Tourniquet Belt & Partially controls bleeding early & $\begin{array}{l}\text { Minimum effectiveness } \\
\text { Maximum mean blood loss } \\
\text { Maximum mean time to } \\
\text { stop bleeding } \\
\text { Minimum percentage of good } \\
\text { composite outcome } \\
\text { Difficult to control bleeding completely } \\
\text { because windlass needs high torque to turn }\end{array}$ \\
\hline ParaBelt & $\begin{array}{l}\text { Minimum mean time } \\
\text { to stop bleeding } \\
\text { Maximum percentage of } \\
\text { good composite outcome } \\
\text { Ratchet is fastest and most } \\
\text { reliable mechanism of } \\
\text { mechanical advantage }\end{array}$ & $\begin{array}{l}\text { Limited tightening before ratchet use } \\
\text { Occasional pinching of skin }\end{array}$ \\
\hline Battle Buddy & $\begin{array}{l}\text { Partially controls } \\
\text { bleeding early }\end{array}$ & $\begin{array}{l}\text { Effectiveness may be dependent } \\
\text { on placing pulley plate over } \\
\text { the femoral artery } \\
\text { Difficult to learn in its specific } \\
\text { technique } \\
\text { Occasional pinching of skin }\end{array}$ \\
\hline
\end{tabular}

and to check performance limitations of the model designs; however, such allowance slowed the expert and impaired performance relative to the cadet. The divergence of strategies was unplanned and inadvertent, but in prior projects with cadets, the expert also has been outperformed by cadets for similar reasons. ${ }^{12,13}$ The importance of user to improved performance of first aid has now been evidenced in multiple studies of varied designs and purposes. ${ }^{8,12-17}$

The second minor finding of the present study was that the evidence of partial hemorrhage control early after beginning application of a tourniquet caused a paradoxical result. Although blood loss and time seemed mismatched at first, the explanation became clear when the rate of blood loss was understood to be a calculated average over time and not an instantaneous measure. Mean rates of blood loss during the time to stop bleeding ranged by model from 4 to $7 \mathrm{~mL} / \mathrm{s}$ for Tourniquet Belt and ParaBelt, respectively. The small differences (eg, 15 $\mathrm{mL}$, or $4 \%$ [15/348]) in mean blood loss seemed paradoxical to the large differences in mean time to stop bleeding (eg, 29 seconds or 34\% [29/85]), but the explanation lies in the differential capability by model to partially control bleeding before achieving full control. For example, Tourniquet Belt had a significantly longer time to stop bleeding than ParaBelt, but this difference is not directly proportional to their difference in blood loss volume because Tourniquet Belt could be tightened faster (before using the windlass) to a greater pressure than ParaBelt could be tightened before its mechanical advantage was used. Therefore, although both models achieved high effectiveness percentages, ParaBelt did not achieve early partial bleeding control like Tourniquet Belt.

As outdoor enthusiasts and wilderness medicine caregivers may consider improvised tourniquets instead of belt tourniquets, a review of pertinent science may inform the decision. The record of improvised tourniquet use is mixed, but the preponderance of data indicates that improvised tourniquets are not reliably effective. The United States Army experience in training student medics users in the Department of Combat Medic Training of the US Army Medical Department's Center and School made an extensive effort to train users in improvised tourniquets after September 11, 2001, but trainers assessed improvised tourniquets as being unreliable in that they could not be 
made reliably effective in the hands of student medics. ${ }^{17}$ The present investigators, who also work for the United States Army, found in 3 separate manikin experiments that improvised tourniquets were unreliable even in the hands of experts. ${ }^{15,17,18}$ In a large cohort of war casualties in 2006 from Baghdad, a survey found that improvised tourniquets were mostly (67\%) ineffective and led to multiple morbidities. ${ }^{19}$ First responders to the bombing at the 2014 Boston Marathon found that improvised tourniquets often were ineffective because they were venous tourniquets and eventually lost control of bleeding. ${ }^{20}$ Despite varied purposes, settings, and people, the experience of the Army medics in training, the investigators in tourniquet research, and caregivers in Baghdad and Boston all found coherent results that indicate that improvised tourniquets are challenging to use well and are unreliable in hemorrhage control. ${ }^{17}$

Use of improvised tourniquets risks partial control of limb bleeding because they commonly act as a venous tourniquet in that the device blocks outflow of blood in compressed veins whose lumens are occluded while inflow through open arteries is ongoing. ${ }^{19}$ Although the first several minutes of such venous tourniquets are relatively well tolerated in normal persons, loss of blood from the core of casualties with each pulsation sequentially worsens hemorrhagic shock. ${ }^{21}$ If the situation is not corrected promptly, the patient has increased risk of foreseeable problems that accrue in orderly increments. Such problems include 1) pooling of blood in distal veins that become distended and engorged, resulting in venous hypertension; 2) expanding wound hematomas with increased need for surgical debridement; 3) loss of plasma from the circulating blood into the local tissues, resulting in edema and distal limb swelling; 4) increased pressure in distal tissues, risking compartment syndrome of limbs, muscle necrosis, and need for surgical fasciotomy to relieve the pressure; 5) more bleeding, which is often paradoxically worse than that without tourniquet use; and 6) given greater pressures in the veins and in the limb, wound bleeding is more difficult to control with compressive methods because pressures applied with such methods have less effect. ${ }^{22}$ Although improvised tourniquets are inferior to well-designed tourniquets, improvised tourniquets may be better than no tourniquet at all in control of difficult bleeding. ${ }^{19}$ At this point, the science so far indicates that the 2 tools, improvised tourniquets and belt tourniquets, need not be an either-or choice, but improvised tourniquets should be a choice of last resort.

Physicians from the United Kingdom in 2015 published a useful article addressing the topic of improvised tourniquet use. ${ }^{23}$ In this article, Stewart et al reviewed such use and cited 36 references. Stewart et al argued a case for improvised tourniquet use and emphasized widespread training of laypersons in the military and civilians as part of both basic life support and emergency caregiving protocols. Their publication helped legitimize improvised tourniquets as a topic of scientific inquiry in part because of both the plain need to stop bleeding almost anywhere and the lack of adequate knowledge of how to reliably do so. Stewart et al evidenced that lack of adequately established knowledge with the few references they could cite that actually reported generated knowledge regarding improvised tourniquets. Improvised tourniquet science previously had been mostly deduction and opinion. Stewart et al explained their technique of improvised use in writing and also illustrated their 5 steps with sequential and clear images. Stewart el al strongly pointed out that the technique of use must be correct in order to both stop bleeding and minimize risk of morbidity and complications. Subsequent to their publication, 3 empiric studies challenged the premise of how reliable users are in improvising tourniquets, and experimental data have indicated that performance is unreliable. ${ }^{17,18,24}$ In the past, it was fairly common for first aid manuals to recommend procedures such as improvised tourniquets and manual compression, but recent trends show occasional refinement of such recommendations to incorporate new evidence of the reliability of users or new evidence of alternatives like commercial tourniquets. Invention of belt tourniquets arose from the necessity to improve on improvised tourniquets.

Limitations of the present study stem mainly from its design: a preliminary experiment in a laboratory. The manikin offers simulation, but it is neither live tissue nor healthcare. There were only 2 evaluators, both of whom had different familiarity with the manikin and the models of tourniquets. The study was not a field assessment of user preference, and the conditions were not stressful. Differential performance was compared with neither improvised tourniquets nor nonbelt tourniquets.

A limitation of the present report stems from a user error. Although the study was designed as a controlled experiment in a laboratory, the expert-the most experienced investigator-made a mistake. The lower mean pressure with Tourni-belt use was the result of user error in forgetting a trait of the manikin. After ending the test, the software program of the manikin samples the pressure during a time period when the touchscreen is blank, although all other data are sampled previously at the time of pressing the finish button on the touchscreen. One user, the expert, inadvertently let the windlass unwind and release the tension in the strap during this interval, causing the 
pressures for Tourni-belt to be lower early in his data collection until this problem was identified and corrected without recurrence. Despite this user's error, the involved model still performed reasonably well overall.

Future directions of study include assessment of refined designs of belt tourniquets, evaluation among more users of varied skill and experience levels, or testing in settings more representative of prehospital care than a laboratory study. A study of the time it takes to remove the belts from the pants may be useful because models may vary. The study of treatment performance should include a focus on the user of the treatment, such as on variation of treatment strategies among users, user learning, and user skill acquisition and maintenance. We now consider user development to be a research priority in first aid science. Comparison studies with non-belt tourniquets, such as standard issue military tourniquets or improvised tourniquets, may show how well varied models and designs work. Perhaps a cadaver model or a live tissue model may provide performance data for different models of belt tourniquet. Later, a human volunteer study may be useful to differentiate models that performed well in prior comparisons.

\section{Conclusions}

In a preliminary analysis of belt tourniquet models in a laboratory setting using a manikin, performance differed by model. All 4 models performed well, such as in their effectiveness, and thus are suitable for further development, such as refinements in device design and their potential for follow-on assessments of user preference during use in the field. ParaBelt had good results in $75 \%$ of tests, which was significantly better than the other models.

Acknowledgment: Lingamanaidu V. Ravichandran of the United States Army Institute of Surgical Research aided in manuscript preparation.

Author Contributions: Study concept and design (JFK, BWB, JKA); acquisition of the data (JFK, BWB); analysis of the data (JFK, JKA); drafting of the manuscript (JFK, BWB, JKA); critical revision of the manuscript (JFK, JKA); and approval of final manuscript (JFK, JKA).

Financial/Material Support: This project was funded by United States Army Medical Research and Materiel Command and the Defense Health Program (Proposal 201105: Operational system management and postmarket surveillance of hemorrhage control devices used in medical care of US Servicepersons in the current war).

Disclosures: None.

Disclaimer: The opinions or assertions contained herein are the private views of the authors and are not to be construed as official or as reflecting the views of the Department of Defense or US Government. The authors are employees of the US Government. This work was prepared as part of their official duties and, as such, there is no copyright to be transferred.

\section{References}

1. Holcomb JB, McMullin NR, Pearse L, et al. Causes of death in US Special Operations Forces in the global war on terrorism: 2001-2004. Ann Surg. 2007;245:986-991.

2. Kragh JF Jr, Beebe DF, O'Neill ML, et al. Performance improvement in emergency tourniquet use during the Baghdad surge. Am J Emerg Med. 2013;31:873-875.

3. Kragh JF Jr, Nam JJ, Berry KA, et al. Transfusion for shock in US military war casualties with and without tourniquet use. Ann Emerg Med. 2015;65:290-296.

4. Beekley AC, Sebesta JA, Blackbourne LH, et al. Prehospital tourniquet use in Operation Iraqi Freedom: effect on hemorrhage control. J Trauma. 2008;64:S28-S37.

5. Andersen RC, Shawen SB, Kragh JF Jr, et al. Extremity War Injuries VII Special Topics Panel. Special Topics. J Am Acad Orthop Surg. 2012;20:S94-S98.

6. Kragh JF Jr, Walters TJ, Baer DG, et al. Survival with emergency tourniquet use to stop bleeding in major limb trauma. Ann Surg. 2009;249:1-7.

7. Kragh JF Jr, Littrel ML, Jones JA, et al. Battle casualty survival with emergency tourniquet use to stop limb bleeding. J Emerg Med. 2011;41:590-597.

8. Kragh JF Jr, O’Neill ML, Walters TJ, et al. The Military Emergency Tourniquet Program's lessons learned with devices and designs. Mil Med. 2011;176:1144-1152.

9. Kragh JF Jr, Walters TJ, Westmoreland T, et al. Tragedy into drama: an American history of tourniquet use in the current war. J Spec Oper Med. 2013;13:5-25.

10. Kragh JF Jr, Burrows S, Wasner C, et al. Analysis of recovered tourniquets from casualties of Operation Enduring Freedom and Operation New Dawn. Mil Med. 2013; 178:799-805.

11. US Food and Drug Administration (FDA). Regulations. Available at: https://www.accessdata.fda.gov/scripts/cdrh/ cfdocs/cfcfr/cfrsearch.cfm?fr=878.5900. Accessed July 8, 2016.

12. Clumpner BR, Polston RW, Kragh JF Jr, et al. Single versus double routing of the band in the Combat Application Tourniquet. J Spec Oper Med. 2013;13:34-41.

13. Polston RW, Clumpner BR, Kragh JF Jr, Jones JA, Dubick MA, Baer DG. No slackers in tourniquet use to stop bleeding. J Spec Oper Med. 2013;13:12-19.

14. Kragh JF Jr, Wallum TE, Aden JK 3rd, Dubick MA, Baer DG. Emergency tourniquet effectiveness in four positions on the proximal thigh. J Spec Oper Med. 2014;14:26-29.

15. Kragh JF Jr, Wallum TE. Aden JK 3rd, Dubick MA, Baer DG. Which improvised tourniquet windlasses work well and which ones won't? Wilderness Environ Med. 2015;26:401405.

16. Davinson JP, Kragh JF Jr, Aden JK 3rd, DeLorenzo RA, Dubick MA. Laboratory testing of emergency tourniquets exposed to prolonged heat. J Spec Oper Med. 2015;15:34-38.

17. Altamirano MP, Kragh JF Jr, Aden JK 3rd, Dubick MA. Role of the windlass in improvised tourniquet use on a manikin hemorrhage model. J Spec Oper Med. 2015;15: 42-46. 
18. Lyles WE 3rd, Kragh JF Jr, Aden JK 3d, Dubick MA. Testing tourniquet use in a manikin model: two improvised techniques. J Spec Oper Med. 2015;15:21-26.

19. Kragh JF Jr, Walters TJ, Baer DG, et al. Practical use of emergency tourniquets to stop bleeding in major limb trauma. J Trauma. 2008;64(Suppl 2):S38-S49. discussion S49-50.

20. King DR, Larentzakis A, Ramly EP, Boston Trauma Collaborative, Tourniquet use at the Boston Marathon bombing: lost in translation. J Trauma Acute Care Surg. 2015;78:594-599.

21. Kragh JF, O’Neill ML, Walters TJ, et al. Minor morbidity with emergency tourniquet use to stop bleeding in severe limb trauma: research, history, and reconciling advocates and abolitionists. Mil Med. 2011;176:817-823.

22. Kragh JF Jr. Use of tourniquets and their effects on limb function in the modern combat environment. Foot Ankle Clinics N Am. 2010;15:23-40.

23. Stewart SK, Duchesne JC, Khan MA. Improvised tourniquets: obsolete or obligatory? J Trauma Acute Care Surg. 2015;78:178-183.

24. Kragh JF Jr, Wallum TE, Aden JK 3rd, Dubick MA, Baer DG. Which improvised tourniquet windlasses work well and which ones won't? Wilderness Environ Med. 2015;26:401-405. 